\title{
ARTICLE
}

Epidemiology

\section{Genetically predicted physical activity levels are associated with lower colorectal cancer risk: a Mendelian randomisation study}

\author{
Xiaomeng Zhang ${ }^{1}$, Evropi Theodoratou ${ }^{1,2}$, Xue Li ${ }^{1,3}$, Susan M. Farrington ${ }^{4}$, Philip J. Law ${ }^{5}$, Peter Broderick ${ }^{5}$, Marion Walker ${ }^{4}$, \\ Yann C. Klimentidis ${ }^{6}$, Jessica M. B. Rees ${ }^{7}$, Richard S. Houlston ${ }^{5}$, lan P. M. Tomlinson ${ }^{2}$, Stephen Burgess $\mathbb{1}^{8}$, Harry Campbell ${ }^{1}$, \\ Malcolm G. Dunlop (10) ${ }^{4}$ and Maria Timofeeva (10)
}

\begin{abstract}
BACKGROUND: We conducted a Mendelian randomisation (MR) study to investigate whether physical activity (PA) causes a reduction of colorectal cancer risk and to understand the contributions of effects mediated through changes in body fat. METHODS: Common genetic variants associated with self-reported moderate-to-vigorous PA (MVPA), acceleration vector magnitude PA (AMPA) and sedentary time were used as instrumental variables. To control for confounding effects of obesity, we included instrumental variables for body mass index (BMI), body fat percentage, waist circumference and arm, trunk and leg fat ratios. We analysed the effect of these instrumental variables in a colorectal cancer genome-wide association study comprising 31,197 cases and 61,770 controls of European ancestry by applying two-sample and multivariable MR study designs.

RESULTS: We found decreased colorectal cancer risk for genetically represented measures of MVPA and AMPA that were additional to effects mediated through genetic measures of obesity. Odds ratio and $95 \%$ confidence interval (Cl) per standard deviation increase in MVPA and AMPA was $0.56(0.31,1.01)$ and $0.60(0.41,0.88)$, respectively. No association has been found between sedentary time and colorectal cancer risk. The proportion of effect mediated through BMI was $2 \%(95 \% \mathrm{Cl}: 0,14)$ and $32 \%(95 \% \mathrm{Cl}$ : $12,46)$ for MVPA and AMPA, respectively.

CONCLUSION: These findings provide strong evidence to reinforce public health measures on preventing colorectal cancer that promote PA at a population level regardless of body fatness.
\end{abstract}

British Journal of Cancer (2021) 124:1330-1338; https://doi.org/10.1038/s41416-020-01236-2

\section{BACKGROUND}

Colorectal cancer is one of the most common cancers of developed societies. ${ }^{1}$ Obesity is one of the risk factors for colorectal cancer. ${ }^{2}$ Ready access to high-calorie foodstuffs combined with a sedentary lifestyle means that obesity has become a major public health problem in developed countries, further contributing to increased colorectal cancer incidence.

Evidence from observational epidemiological studies is consistent with the premise that increased physical activity (PA) reduces colorectal cancer risk. Furthermore, the magnitude of the effect is sufficient to be meaningful for the individual. ${ }^{3}$ An umbrella review of 22 anatomical cancer sites concluded that there is strong evidence for a protective association between self-reported recreational PA and colorectal cancer. ${ }^{4}$ In addition, a recent meta-analysis of 17 cohorts and 21 case-control studies found that occupational activity, recreational activity, transport-related PA and reduced occupational sedentary behaviour were each associated with lower colorectal cancer risk. ${ }^{5}$ The estimated effects of increased recreational PA and occupational sedentary behaviour for colon cancer risk were 0.80 (95\% confidence interval (Cl): $0.71,0.89)$ and 1.44 (95\% Cl: 1.28, 1.62), respectively, and for rectal cancer risk were 0.87 (95\% Cl: $0.75,1.01)$ and $1.02(95 \% \mathrm{Cl}: 0.82,1.28)$ respectively. ${ }^{5}$ Despite the strength of such correlative evidence on the effect of PA on colorectal cancer risk, causality cannot be ascribed by observational studies, since the observed association could be due to confounding factors or residual confounding. Furthermore, PA is routinely measured in observational studies as a self-reported activity, and this may be systematically overestimated. ${ }^{6}$

Given the lack of randomised clinical trials (RCTs) to formally test the effect of a PA intervention on colorectal cancer risk, one

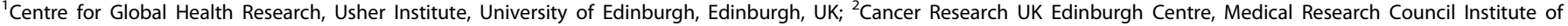

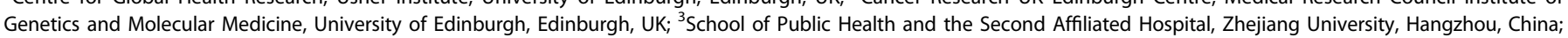

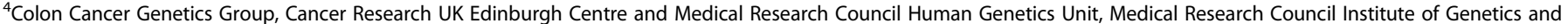

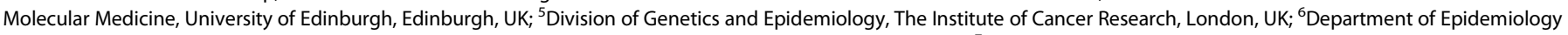

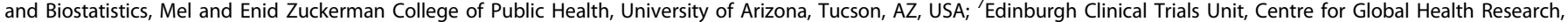

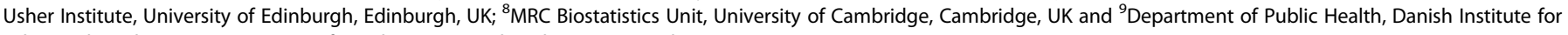
Advanced Study (DIAS), University of Southern Denmark, Odense, Denmark
}

Correspondence: Malcolm G. Dunlop (malcolm.dunlop@ed.ac.uk) or Maria Timofeeva (mtimofeeva@health.sdu.dk)

These authors contributed equally: Xiaomeng Zhang, Evropi Theodoratou

Received: 4 August 2020 Revised: 20 November 2020 Accepted: 8 December 2020

Published online: 29 January 2021 
approach is to apply Mendelian randomisation (MR) approaches to test whether the association is causal. MR explores the effect of the exposure (PA) on colorectal cancer risk through a genetic instrumental variable. ${ }^{7}$ Since the instrumental variable is randomly assorted at conception, it can overcome the aforementioned shortcomings such as confounding effects. Common genetic variants shown in genome-wide association studies (GWASs) to be associated with PA can be used as instrumental variables for various measures of PA. One recent MR study supports a causal association between higher PA measured by accelerometer and lower colorectal cancer risk. ${ }^{8}$ However, potential confounding or mediating effect of body fatness was not taken into account. ${ }^{8}$

As the most commonly used measure of body fatness, body mass index (BMI) is often considered as a proxy of overall body fat. The body fat percentage measured by bioimpedance is another proxy of overall body fat to compare with the results from BMI. However, for people with the same overall body fat (i.e. BMI and body fat percentage), body fat distribution changes with factors such as sex, age, ethnicity, nutritional status and fitness training level. ${ }^{9}$ Evidence shows that people with normal BMI but excess trunk fat are at higher risk of metabolic diseases, ${ }^{10}$ while those with a normal BMI but with excess leg fat are at lower myocardial infarction risk. ${ }^{11}$ The effect of excess body fat (BMI, body fat percentage, waist circumference and body fat distribution) on colorectal cancer risk has been well described in observational ${ }^{2}$ and MR studies. ${ }^{12,13}$

Here, using MR approaches, we have investigated the observed association between PA and colorectal cancer to establish, or refute, causality. Further, we have tested whether the effects are confounded by, or mediated through, measures of body fat. In order to comprehensively assess the influence of body fat, we employed measures of body fat including BMI, body fat percentage, waist circumference and three types of body fat distribution measured by bioimpedance.

\section{METHODS}

Genotype data resources for colorectal cancer case-control genome-wide association analysis

We used genome-wide summary-level genotyping data imputed to a merged reference panel comprising the 1000 Genome Project and UK10K from a meta-analysis of 15 GWAS datasets ${ }^{14}$ from populations of European ancestry (Supplementary Method and Table S1). Briefly, the colorectal cancer GWAS meta-analysis included the following GWASs: NSCCG, the SCOT study, SOCCS/
GS, SOCCS/LBC and UK Biobank GWAS, as well as ten previously published GWASs: UK1, Scotland1, VQ58, CCFR1, CCFR2, COIN, Finnish GWAS, CORSA, DACHS and Croatia. Standard qualitycontrol measures were applied to each GWAS and summary statistic data from 31,197 cases and 61,770 controls were included in the analyses. ${ }^{14}$ GWAS data from the UK Biobank was excluded from the sensitivity analysis to avoid bias caused by sample overlap between exposure and outcome datasets. All studies were approved by respective ethics/institutional review committees, in accordance with the Declaration of Helsinki. Participants of all the included studies have signed the relevant consent forms.

\section{Generation of genetic instruments}

We implemented genetic instrumental variables for three validated measures of continuous PA: self-reported moderate-tovigorous PA (MVPA), overall acceleration vector magnitude PA (AMPA) and sedentary time. Instrumental variables for PA were extracted from two GWASs that established associations between common genetic variants (minor allele frequency $\geq 5 \%$ ) and PA (Table S2): (i) a meta-analysis of GWASs of 337,234 UK Biobank participants on habitual PA; ${ }^{15}$ (ii) a GWAS of average acceleration vector magnitude on 91,105 UK Biobank participants. ${ }^{16}$ All summary-level statistics from the GWASs used in the current work were restricted to CEU (northern and western European) populations. Therefore, population stratification is not a potential bias in our study. Data for the MVPA measure were derived from self-reported questionnaires completed as part of the UK Biobank dataset collected between 2006 and 2010. ${ }^{17}$ AMPA and sedentary time were collected from a subset of 91,105 UK Biobank participants wearing an accelerometer 7 days between 2013 and $2015 .^{18}$ Details of MVPA, AMPA and sedentary time were described in the Supplementary Methods. Klimentidis et al. ${ }^{15}$ detected eight single-nucleotide polymorphism (SNPs) for MVPA at $P<5 \times 10^{-9}$ (Table 1). Doherty et al. ${ }^{16}$ detected three SNPs at $P<5 \times 10^{-9}$ and five SNPs at $P<5 \times 10^{-8}$ for AMPA and six SNPs for the sedentary time at $P<5 \times 10^{-8}$ (Table 1). The SNP-based heritability estimate was $5 \%$ for MVPA, $21 \%$ for AMPA and $12.9 \%$ for sedentary time. ${ }^{15,16}$

Several genetic variants associated with MVPA, AMPA and sedentary time were previously found to be also associated with the weight, BMI and arm/body/leg/trunk fat percentage (Table S3). We included BMI, body fat percentage, waist circumference and body fat distribution as indicators of obesity in our analysis. The body fat distribution consists of arm fat ratio (AFR), trunk fat ratio (TFR) and leg fat ratio (LFR) measured by segmental bio-electrical

Table 1. Description of instrumental variable (IV) for each measure.

\begin{tabular}{|c|c|c|c|c|c|}
\hline Exposures & $\begin{array}{l}\text { No. of SNPs } \\
\text { for the IV }\end{array}$ & $\begin{array}{l}\text { Variance explained } \\
\text { by the IV }\left(R^{2}\right)^{\mathrm{a}}\end{array}$ & $\begin{array}{l}\text { Sample size of } \\
\text { exposure GWAS }\end{array}$ & Population & Reference (PMID) \\
\hline MVPA & 7 & 0.0007 & 385,790 & European & Klimentidis et al. ${ }^{15}$ (29899525) \\
\hline AMPA & 5 & 0.002 & 91,105 & European & Doherty et al. ${ }^{16}$ (30531941) \\
\hline AMPA $^{\mathrm{b}}$ & 3 & 0.001 & 91,105 & European & Doherty et al. ${ }^{16}$ (30531941) \\
\hline Sedentary time & 6 & 0.001 & 91,105 & European & Doherty et al. ${ }^{16}$ (30531941) \\
\hline BMI & 68 & 0.0232 & 322,154 & European & Locke et al. ${ }^{60}(25673413)$ \\
\hline Body fat percentage & 370 & 0.053 & 331,117 & European & Bycroft et al. ${ }^{61}$ (30305743) \\
\hline Waist circumference & 59 & 0.010 & 224,459 & European & Shungin et al. ${ }^{62}$ (25673412) \\
\hline AFR & 15 & 0.002 & 362,499 & European & Rask-Andersen et al. ${ }^{11}$ (30664634) \\
\hline TFR & 50 & 0.002 & 362,499 & European & Rask-Andersen et al. ${ }^{11}$ (30664634) \\
\hline LFR & 46 & 0.002 & 362,499 & European & Rask-Andersen et al. ${ }^{11}$ (30664634) \\
\hline
\end{tabular}

GWAS genome-wide association study, MVPA self-reported moderate-to-vigorous physical activity, AMPA acceleration vector magnitude physical activity, SNP single-nucleotide polymorphism, IV instrumental variable, BMI body mass index, AFR arm fat ratio, LFR leg fat ratio, TFR trunk fat ratio.

${ }^{\mathrm{a}} R^{2}$ was estimated base on the formula: $2 \times \mathrm{EAF} \times(1-\mathrm{EAF}) \times \beta^{2}$.

'Threshold at $P<5 \times 10^{-9}$. 

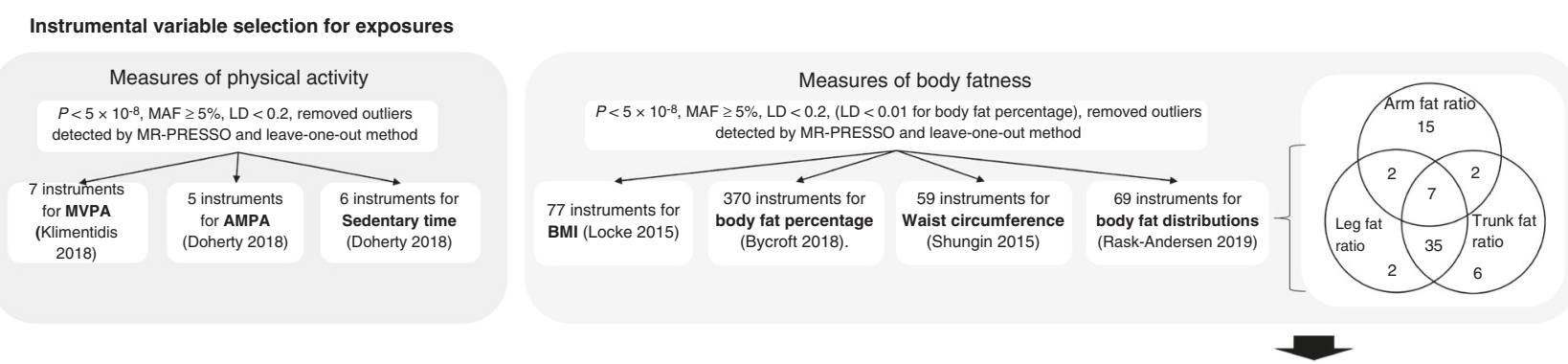

Two-sample Mendelian randomisation analysis: effects of physical activity and measures of body fatness on colorectal cancer risk

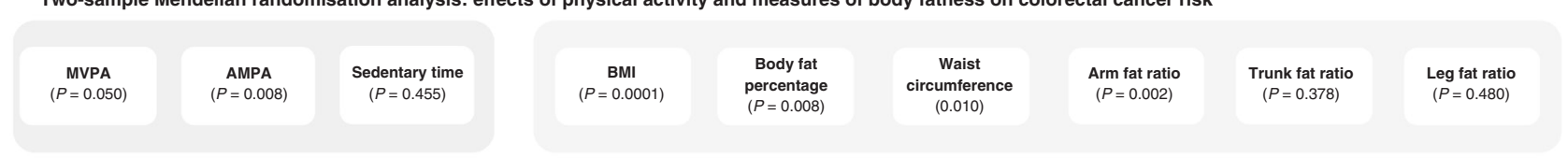

Multivariable Mendelian randomisation analysis: effects of multiple exposures on colorectal cancer risk

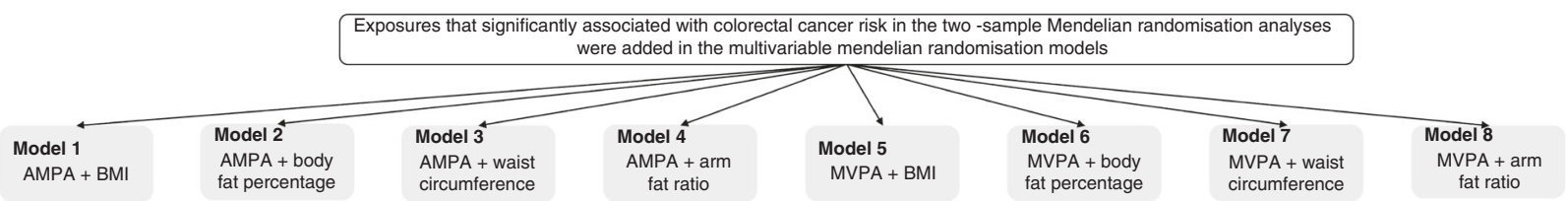

Fig. 1 Schematic representation of the study design. MAF minor allele frequency, LD linkage disequilibrium, MVPA self-reported moderateto-vigorous physical activity, AMPA acceleration vector magnitude physical activity, BMI body mass index.

impedance (sBIA). The information about instrumental variables for measures of body fatness can be found in Supplementary Method, Table 1 and Fig. 1. The threshold of linkage disequilibrium (LD) was set as $R^{2}>0.2$. Due to the limited variance explained by the MVPA SNPs, and the fact that the inverse variance-weighted, MR-Egger and MR-Robust methods can incorporate correlation between variants, we included all the SNPs to generate instrumental variable and added correlation matrix into these analyses (available in R package 'MendelianRandomization'). We excluded rs149943 from the median-based method because of LD $\left(r^{2}=0.35\right)$ with rs3094622.

\section{Two-sample MR}

Having determined the effect estimates of SNPs on PA and each measure of body fat from GWASs (Table 1), we tested the effects of these SNPs on colorectal cancer risk using genome-wide data from our previous colorectal cancer GWAS meta-analysis. ${ }^{14}$ The causal effects and the corresponding standard errors of exposures on colorectal cancer were calculated by using the random-effect inverse variance-weighted method. ${ }^{19}$ We then evaluated the heterogeneity among the causal effects of each variant (Cochran's $Q$ statistic). $P$ values $<0.10$ were considered indicative of significant heterogeneity. ${ }^{20}$

\section{Multivariable MR}

We applied multivariable $M R^{21}$ to elucidate the causal relationship between PA and colorectal cancer while keeping measures of body fatness constant. First, we analysed the beta-beta correlations of SNP effects for each exposure through Pearson's correlation coefficient analysis, including significant SNPs of all measures of PA and body fatness. Next, we ran multivariable MR for measures of PA that significantly associated with colorectal cancer risk in the two-sample MR by adding measures of body fatness that significantly associated with colorectal cancer risk in the twosample MR as covariates. In total, eight models were fitted (Fig. 1). All SNPs associated with each trait were included to generate instrumental variables for each model (Fig. 1). The pairwise LD threshold between all of these SNPs was set at $R^{2}>0.2$.

The rationale of the study design is shown in Fig. 1 .
Sensitivity analysis

We applied a variety of sensitivity analyses testing different MR assumptions. $^{22}$ Specifically, we performed MR-Pleiotropy Residual Sum and Outlier (MR-PRESSO), ${ }^{23} \mathrm{MR}^{2}$ Robust, ${ }^{24}$ MR-Egger, ${ }^{25}$ leaveone-out method, ${ }^{26}$ mode-based estimate ${ }^{27}$ and the median-based method. ${ }^{28}$ MR-Robust applies MM-estimation (modified maximum-likelihood estimation) with Tukey's bisquare function, which efficiently limits the contribution of outliers. ${ }^{24}$ MR-Egger was applied to explore any potential bias introduced by pleiotropy. In particular, when the intercept of MR-Egger differs from zero (at $p<0.05$ ), then either directional pleiotropy is indicated or the InSIDE assumption is violated. ${ }^{25}$ We also applied the mode-based estimate, which works well when most estimates of identical individual-instrument causal effects are derived from valid instrumental variables and the weighted median-based method, which allows for $50 \%$ of invalid weights. ${ }^{27,28}$

MR-PRESSO was applied to identify horizontal pleiotropic outliers. $^{23}$ When both MR-PRESSO and leave-one-out method indicated an outlier, we took the analysis after removing the outlier as our main analysis. Two-sample MR estimates can be biased when samples between exposure and outcome GWASs overlapped. ${ }^{29}$ To minimise the risk of this bias, we performed sensitivity analysis after excluding UK Biobank cohort participants from colorectal cancer GWAS meta-analysis. In addition, we checked the GWAS Catalogue ${ }^{30,31}$ (https://www.ebi.ac.uk/gwas/home, accessed on 2 February 2020) and PhenoScanner ${ }^{32,33}$ (http://www. phenoscanner.medschl.cam.ac.uk/, accessed on 2 February 2020) to determine whether the PA instrumental variables were associated with other traits, consistent with pleiotropic effects. For all MR analyses, the $P$ value threshold was set at 0.05 . All statistical analyses were performed on $\mathrm{R}$ v3.6.1 with packages 'MendelianRandomization' and 'TwoSampleMR'. ${ }^{26,34}$

Power calculation

The non-centrality parameter-based approach was applied to estimate the power of this study. ${ }^{35}$ The $R^{2}$ (the variance explained by each genetic instrument) was estimated by the following formula: $2 \times \mathrm{EAF} \times(1-\mathrm{EAF}) \times \beta^{2}$ and F-statistic $F=R^{2} \times(N-$ $2) /\left(1-R^{2}\right)$ was applied to estimate the strength of genetic 


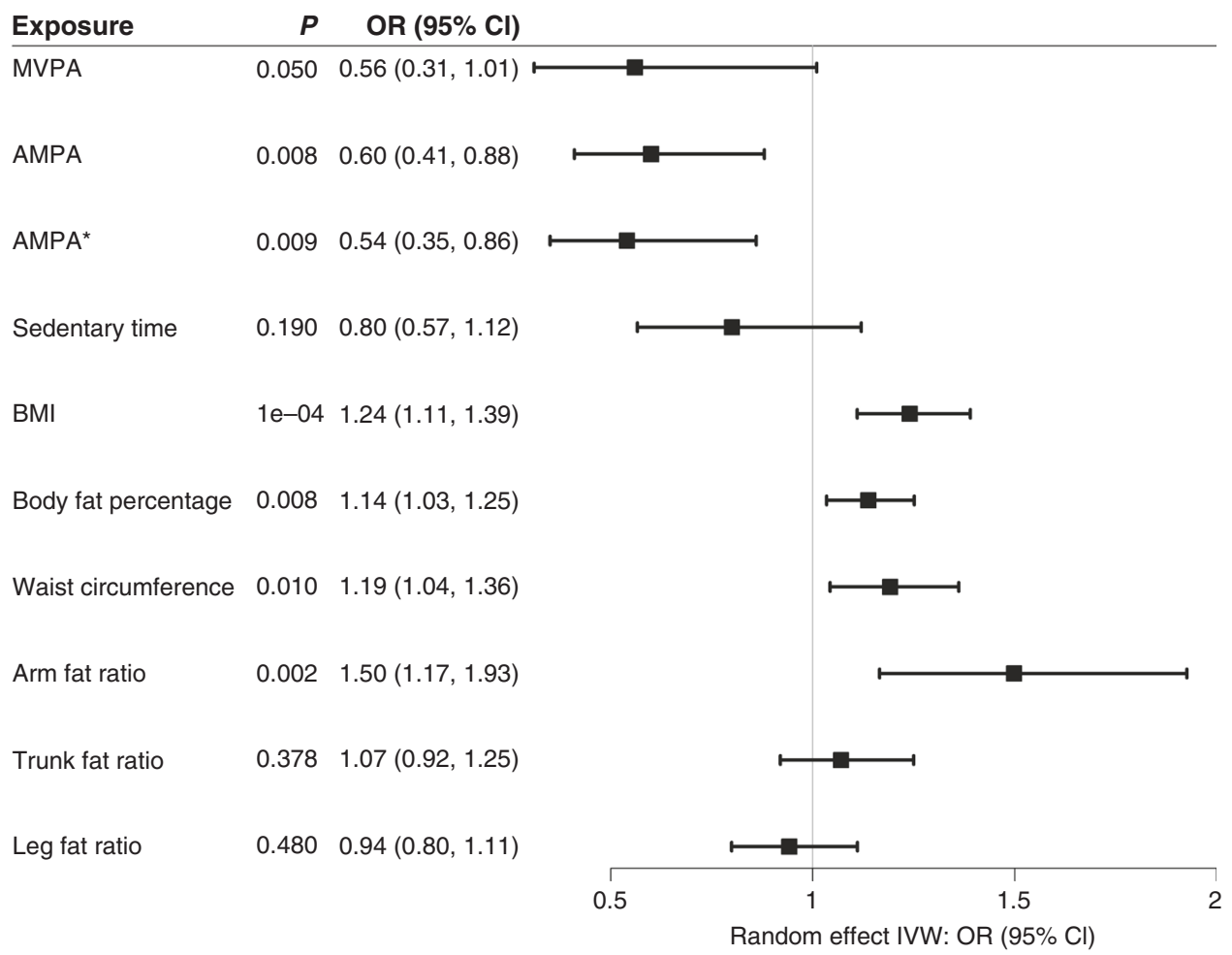

Fig. 2 Forest plot of two-sample Mendelian randomisation studies exploring associations between exposures to colorectal cancer risk. MVPA self-reported moderate-to-vigorous physical activity, AMPA acceleration vector magnitude physical activity at $P<5 \times 10^{-8}$, AMPA $^{*}$ acceleration vector magnitude physical activity at $P<5 \times 10^{-9}$, BMI body mass index, OR odds ratio, Cl confidence interval, IVW inverse variant-weighted method, $P P$ value for random effect IVW result.

instrument, ${ }^{36,37}$ where EAF is effect allele frequency, beta is the effect size of instrumental variables per standard deviation (SD) change of PA and $N$ is the sample size of PA GWAS. The MVPA-, AMPA- and sedentary time-related variants explained $\sim 0.07 \%$, $0.2 \%$ and $0.1 \%$ of the phenotypic variance, respectively. We fixed the type I error at $a<0.05$ and listed the effect estimates that could be detected for each SD increase of the PA time. We required $80 \%$ power to detect any effects. Effect sizes that can be detected with the power of 0.8 , as well as F-statistics for instrumental variables are presented in Table S4. The F-statistic for all the analyses did not indicate weak instruments $(F>10)$.

\section{RESULTS}

Two-sample MR

The MR result indicated a decreased colorectal cancer risk through the effect of MVPA. The odds ratio (OR) for inverse varianceweighted MR was 0.56 for colorectal cancer risk per 1 SD increase of MVPA (95\% Cl: $0.31,1.01$ ) (Figs. 2 and S1) and each sensitivity analysis method generated similar effect estimates (Table S5). The intercept of MR-Egger regression test did not identify any horizontal pleiotropy and/or violation of the InSIDE assumption $(P=0.75)$ and the $Q$-statistic did not indicate heterogeneity $(P=$ 0.56). After removing the UK Biobank data case-control study (4800 cases and 20,289 controls) from the outcome population, the $\mathrm{Cl}$ of effect sizes was wider (Table S5).

Implementing the MR-PRESSO and leave-one-out methods highlight rs429358 as an outlier (Fig. S2). This SNP maps to the APOE gene and showed the strongest association with PA. It is also associated with multiple traits, including total cholesterol, low-density lipoprotein cholesterol, triglyceride and Alzheimer's disease. ${ }^{38,39}$ This finding was supported by searching from GWAS Catalogue and PhenoScanner (Table S3). Therefore, we generated the instrumental variable by using seven SNPs for MVPA after removing rs429358. The effect sizes of the seven MVPA SNPs with MVPA and with colorectal cancer were presented in Table S6.

Evidence for a causal association was detected between AMPA and colorectal cancer risk by using both five SNPs at $P<5 \times 10^{-8}$ and three SNPs at $P<5 \times 10^{-9}$ as genetic instruments (Fig. 2). In particular, the ORs of inverse variance-weighted MR were 0.60 (95\% Cl: $0.41,0.88)$ and 0.54 (95\% Cl: $0.35,0.86)$ for each genetic instrument (Figs. 2 and S1). All sensitivity analyses showed a similar effect size of the association between AMPA and colorectal cancer risk (Table S5). The $Q$-statistic suggested no heterogeneity $(P=0.34$ and 0.35 , respectively) and the intercept of MR-Egger suggested no pleiotropy. Removing UK Biobank colorectal cancer GWAS from the outcome populations narrowed the Cls of the effect sizes (Table S5). No association was detected between sedentary time and colorectal cancer risk and no pleiotropy or heterogeneity was indicated (Fig. 2 and Table S5). The effect estimates for each instrumental variable on AMPA and colorectal cancer or sedentary time and colorectal cancer were listed in Table S6.

Two-sample MR on the associations between measures of body fatness and colorectal cancer risk, as well as the effect estimates of each SNP on exposures were listed in Table S7-10. BMI, body fat percentage, waist circumference and AFR were significantly associated with increased colorectal cancer risk, with OR $(95 \%$ Cl) 1.24 (1.11, 1.39), $1.14(1.03,1.25), 1.19(1.04,1.36)$ and 1.50 (1.17, 1.93), respectively (Fig. 2). We did not observe any significant associations between TFR or LFR and colorectal cancer risk.

Multivariable MR

Instrumental variables for BMI, waist circumference and AFR were highly correlated as their correlation coefficients $(r)$ range from 0.67 to 0.79 (Fig. 3). Body fat percentage was positively correlated with BMI $(r=0.13)$, waist circumference $(r=0.41)$, AFR $(r=0.33)$ and TFR $(r=0.16)$. LFR and TFR were highly inversely 


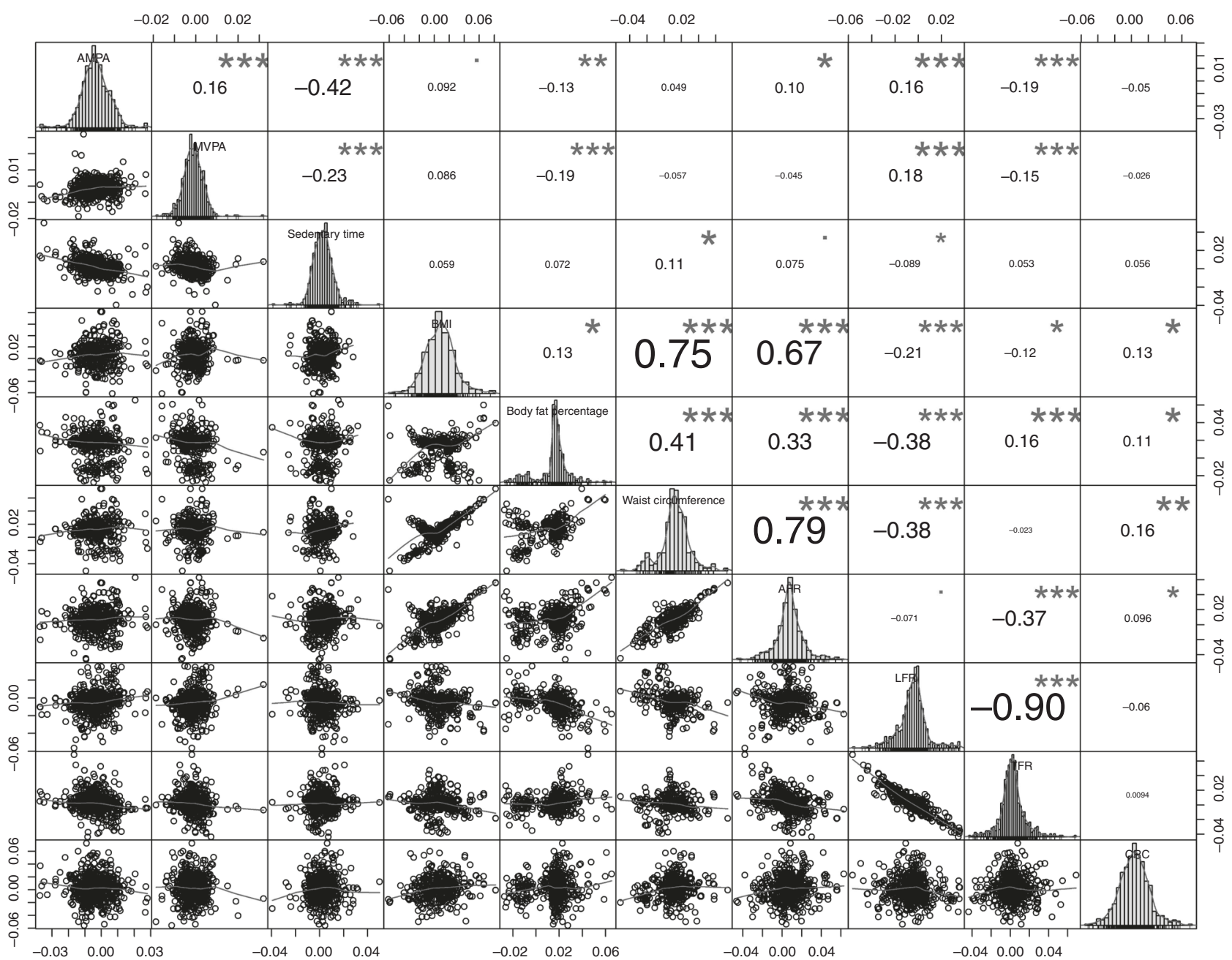

Fig. 3 Genetic correlation coefficient between each covariate. MVPA self-reported moderate-to-vigorous physical activity, AMPA acceleration vector magnitude physical activity, BMI body mass index, CRC colorectal cancer, AFR arm fat ratio, LFR leg fat ratio, TFR trunk fat ratio.

correlated $(r=-0.90)$. MVPA and AMPA were positively correlated with each other $(r=0.16)$ and negatively correlated with sedentary time $(r=-0.23$ and -0.42 respectively). The three measures of PA were not correlated with colorectal cancer risk.

We estimated the direct effect of MVPA and AMPA and four different measures of obesity and overweight on colorectal cancer risk by applying the multivariable MR method (Table 2). The direct effect sizes (OR) of MVPA or AMPA on colorectal cancer when BMI was kept constant were $0.56(95 \% \mathrm{Cl}: 0.36,0.89)$ and $0.70(95 \% \mathrm{Cl}$ : $0.52,0.96)$, respectively. The direct effect sizes (OR) of $\mathrm{BMI}$ on colorectal cancer when MVPA or AMPA was kept constant were 1.24 (95\% Cl: 1.12, 1.37) and 1.22 (95\% Cl: 1.09, 1.36), respectively. There was no statistically significant heterogeneity and MR-Egger did not indicate a significant pleiotropy effect. Compared to the effect estimates from two-sample MR, the estimated Cls of the two types of PA from multivariable MR became much wider, while the BMI effects were not affected (Table 2 and Fig. 2). Results from two-step network MR also indicated a partial mediation effect of $\mathrm{BMI}$ on the PA-colorectal cancer association (Table S11).

The direct effect sizes (OR [95\% CI]) of MVPA or AMPA on colorectal cancer when AFR was kept constant were $0.58(0.33$, $1.02)$ and $0.64(0.45,0.92)$, respectively. The direct effect sizes of AMPA on colorectal cancer when waist circumference was kept constant were $0.61(0.39,0.97)$, while no association observed for MVPA after adjusting for waist circumference. The associations between MVPA or AMPA and colorectal cancer risk disappeared when keeping body fat percentage constant.

Based on the total effect estimates from two-sample MR and direct effect estimates from multivariable MR, we evaluated the proportion of effects of PA on colorectal cancer risk mediated through measures of body fat. The attenuated direct effects indicated that part of the effects of the two measures of PA on colorectal cancer was mediated through BMI or AFR. AMPA also can affect colorectal cancer risk through waist circumference. For the effects of MVPA and AMPA on colorectal cancer risk, the proportion mediated through BMI was $2 \%(95 \% \mathrm{Cl}: 0,14)$ and $32 \%$ (95\% Cl: 12,46$)$, respectively, while the proportion mediated through AFR was $8 \%(95 \% \mathrm{Cl}: 0,16)$ and $14 \%(95 \% \mathrm{Cl}: 0,35)$, respectively (Fig. 4). The proportion of effects mediated through waist circumference was $5 \%(95 \% \mathrm{Cl}: 0,22)$ for AMPA (Fig. 4).

\section{DISCUSSION}

Colorectal cancer is a common cancer with appreciable morbidity and mortality. Prospective cohort, case-control, and crosssectional observational studies support an inverse association between PA and colorectal cancer risk. ${ }^{40-42}$ The association between AMPA and colorectal cancer risk has been reported in a previous MR study. ${ }^{8}$ However, the mechanism(s) through which PA influences colorectal cancer risk is not clear yet, especially the 
Table 2. Results of multivariable Mendelian randomisation analysis: causal estimation of MVPA, AMPA and measures of body fatness on colorectal cancer risk.

\begin{tabular}{|c|c|c|c|c|c|c|c|c|}
\hline & OR $(95 \% \mathrm{Cl})$ & $P$ & $P_{\text {int }}^{a}$ & $P_{\text {het }}^{\mathrm{b}}$ & OR $(95 \% \mathrm{Cl})$ & $P$ & $P_{\mathrm{int}}{ }^{\mathrm{a}}$ & $P_{\text {het }}^{\mathrm{b}}$ \\
\hline \multicolumn{9}{|l|}{ Model 1 (AMPA + BMI) } \\
\hline AMPA & $0.70(0.52,0.96)$ & 0.02 & / & 0.15 & $0.71(0.46,1.10)$ & 0.13 & 0.95 & 0.13 \\
\hline BMI & $1.22(1.09,1.36)$ & $4.65 \mathrm{E}-04$ & & & $1.01(0.83,1.23)$ & 0.001 & & \\
\hline AMPA & $0.86(0.67,1.10)$ & 0.24 & / & $9.74 \mathrm{E}-13$ & $0.83(0.59,1.15)$ & 0.26 & 0.70 & $8.01 E-13$ \\
\hline Body fat percentage & $1.10(0.98,1.24)$ & 0.10 & & & $1.11(0.98,1.25)$ & 0.09 & & \\
\hline \multicolumn{9}{|c|}{ Model 3 (AMPA + waist circumference) } \\
\hline AMPA & $0.61(0.39,0.97)$ & 0.04 & / & 0.05 & $0.61(0.39,0.97)$ & 0.04 & 0.26 & 0.05 \\
\hline Waist circumference & $1.21(1.07,1.36)$ & 0.003 & & & $1.21(1.07,1.36)$ & 0.003 & & \\
\hline MVPA & $0.56(0.36,0.89)$ & 0.01 & / & 0.22 & $0.58(0.29,1.15)$ & 0.12 & 0.91 & 0.19 \\
\hline BMI & $1.24(1.12,1.37)$ & $7.67 E-5$ & & & $1.24(1.11,1.38)$ & $1.32 \mathrm{E}-4$ & & \\
\hline \multicolumn{9}{|c|}{ Model 6 (MVPA + body fat percentage) } \\
\hline MVPA & $0.84(0.56,1.26)$ & 0.41 & / & $3.00 E-12$ & $0.60(0.33,1.06)$ & 0.08 & 0.10 & $5.36 \mathrm{E}-12$ \\
\hline Body fat percentage & $1.12(1.02,1.24)$ & 0.02 & & & $1.13(1.02,1.25)$ & 0.02 & & \\
\hline \multicolumn{9}{|c|}{ Model 7 (MVPA + waist circumference) } \\
\hline MVPA & $0.70(0.42,1.17)$ & 0.18 & / & 0.05 & $0.64(0.30,1.34)$ & 0.24 & 0.73 & 0.05 \\
\hline Waist circumference & $1.19(1.05,1.34)$ & 0.01 & & & $1.20(1.05,1.36)$ & 0.01 & & \\
\hline \multicolumn{9}{|l|}{ Model 8 (MVPA + AFR) } \\
\hline MVPA & $0.58(0.33,1.02)$ & 0.06 & / & 0.50 & $0.44(0.20,0.93)$ & 0.03 & 0.27 & 0.52 \\
\hline
\end{tabular}

relative role of body weight and distribution in this association. Applying two-sample and multivariable MR approaches, we used genetic variants from large GWAS as instruments to explore whether the apparent beneficial effects of three measures of PA (MVPA, AMPA and sedentary time) on colorectal cancer risk are mediated through body fatness. Our results show that both lower MVPA and AMPA increase colorectal cancer risk, both independently and through body fatness. In line with our findings, a metaanalysis has indicated that PA is associated with colorectal cancer risk in both high and low BMI groups. ${ }^{43}$

It is important to note that we found that increasing PA causes decreased colorectal cancer risk independent of measures of obesity and body fat distribution. One SD of MVPA is $\sim 4.96$ metabolic equivalent task (MET)-h/day. MET is an objectively measured ratio of energy expenditure relative to the mass of a person when performing PA compared to sitting quietly. Although there is no standard method to transform milli-gravities (accelerometer measurement units) to energy expenditure, each SD of AMPA was 8.14 milli-gravities (or $0.08 \mathrm{~m} / \mathrm{s}^{2}$ ), which approximates to $3 \mathrm{MET}-\mathrm{h} /$ day. $^{18}$ For each SD increase in MVPA or AMPA, colorectal cancer risk decreased by $44 \%$ and $40 \%$, respectively. These estimates imply that if individuals replace daily sedentary behaviour with 20-90 min of MVPA or with 13-60 min accumulated MVPA, their risk of colorectal cancer will decrease by $40 \%$ (Table S12). ${ }^{44}$ In our study, MVPA estimated a longer time spending on PA compared to the estimation by AMPA to achieve a similar benefit of colorectal cancer risk, which is consistent with the existing evidence that MVPA tends to overestimate time engaged in PA in the general population. ${ }^{45}$ Part of the decreased effect on colorectal cancer risk (2\% [95\% Cl: 0,14$]$ and $8 \%$ [95\% Cl: $0,16]$ for MVPA and 32\% [95\% Cl: 12,46$]$ and $14 \%$ [95\% Cl: 0,35$]$ for AMPA) was mediated through the effects of BMI and AFR, respectively. Since the genetic instrument of MVPA is weaker compare to AMPA, the BMI and AFR mediation effect for MVPA may be underestimated. ${ }^{46}$

Diverse biological mechanisms have been proposed to explain the observed inverse association between PA and colorectal cancer. These include beneficial effects on bowel transit time, ${ }^{47}$ immune system reactions, ${ }^{48}$ metabolisms of bile acid, better insulin sensitivity ${ }^{49}$ and the reduction of prostaglandin E2 levels in colonic mucosa. ${ }^{50}$ Evidence from RCTs supports that PA can reduce the bowel transit time and therefore reduce the time of contact between carcinogens and colonic mucosa. ${ }^{47}$ The decrease of prostaglandin E2 synthesis may also promote intestinal peristalsis and hence reduce transit time. ${ }^{51}$ Besides, prostaglandin E2 can promote tumour generation directly or through its multifaceted effects on inflammation. ${ }^{52}$ PA also results in a lower concentration of bile acid, which is an essential mediator of the cholesterol mechanism and the lower bile acid concentration is associated with lower blood triglycerides. ${ }^{53}$ The effect between PA 


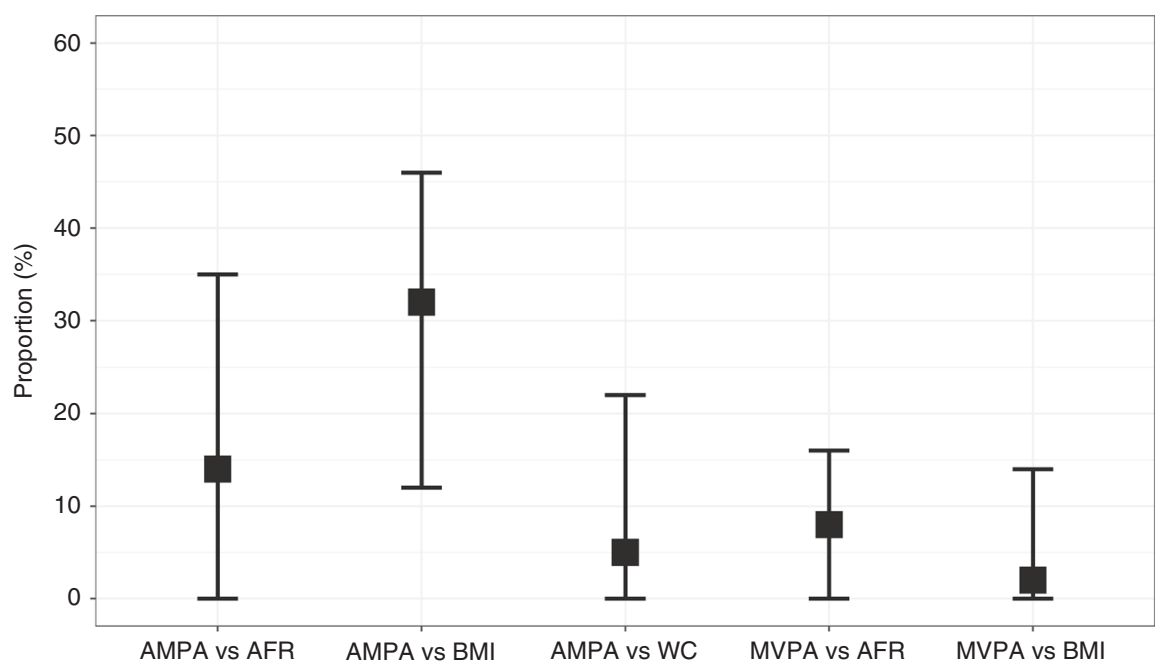

Fig. 4 Proportion estimates through BMI and body fat composition. BMI body mass index, MVPA self-reported moderate-to-vigorous physical activity, AMPA acceleration vector magnitude physical activity, AFR arm fat ratio, WC waist circumference, AMPA vs AFR the proportion of effects of AMPA on colorectal cancer risk mediated through AFR, AMPA vs BMI the proportion of effects of AMPA on colorectal cancer risk mediated through BMI, AMPA vs WC the proportion of effects of AMPA on colorectal cancer risk mediated through WC, MVPA vs AFR: the proportion of effects of AMPA on colorectal cancer risk mediated through AFR, MVPA vs BMI the proportion of effects of AMPA on colorectal cancer risk mediated through BMI.

and colorectal cancer risk could be through these pathways, although none of the genetic variants included as an instrumental variable for PA was located within genes involved in indicators of above-mentioned metabolism pathways. In addition, regular moderate PA may have a benefit on natural cytotoxicity and Tlymphocyte proliferation, on reducing the production of proinflammatory cytokines and on increasing the count of $T$ cells, B cells and immunoglobulins. ${ }^{48}$

Strengths and limitations

One of the strengths of this study was that we explored both subjective and objective measures of PA (MVPA, AMPA and sedentary time). Previous studies showed that there are discrepancies between MVPA and AMPA. ${ }^{54}$ Compared to MVPA where recall and reporting bias are problematic, AMPA explains $44-47 \%$ variance of energy expenditure. ${ }^{55}$ MVPA tends to overestimate time engaged in PA in the general population. ${ }^{45}$ Nevertheless, MVPA is commonly used in epidemiological and observational studies, because it is data that is readily collected and inexpensive. Our results for MVPA and AMPA were consistent, with both supporting a causal effect of PA in reducing cancer risk. The confidence intervals for the effect estimates observed for AMPA were appreciably narrower than for MVPA, suggesting the possibility of recall bias, but the health-promoting effect of actually wearing an accelerometer might also influence our results. ${ }^{56}$ Nonetheless, our use of a variety of instrumental variable methods provides new insight into the effect of PA on cancer risk. Another main advantage of our study is that we have clarified the association pathways among three measures of PA, different measures of body fatness and colorectal cancer for the first time, including the total effect and direct effect, as well as the proportion of the indirect effect. Several measures of body fatness have been considered in our study, BMI and body fat percentage represent two different measures of total body fat while waist circumference, AFR, TFR and LFR represent body fat in different areas. In the two-sample MR study, BMI, body fat percentage, waist circumference and AFR are associated with colorectal cancer risk, which is consistent with the previous evidence. ${ }^{12,57}$ Based on the results of multivariable MR, AMPA can affect colorectal cancer risk both through and independent of $\mathrm{BMI}$, waist circumference and AFR, while MVPA can affect colorectal cancer risk both through and independent of BMI and AFR. After adjusting for body fat percentage, associations between AMPA or MVPA and colorectal cancer risk disappeared, which may be due to the high heterogeneity introduced by using hundreds of SNPs as the instrumental variable. Papdimitriou et al. performed an MR on PA and CRC risk and they also considered the effects of BMI in the sensitivity analysis. In the current study, we have expanded previous work by additionally including genetic instruments for measures of body fatness along with PA in multivariable MR.

We acknowledge that the study has several limitations. First, although we derived instrumental variables from the largest available GWAS for PA, the SNPs for self-reported PA explain only $0.07 \%$ of the variance in MVPA. However, the calculated F-statistic $(F$-statistic $=273)$ reached a widely-accepted threshold level. ${ }^{37}$ As a result, our analysis of MVPA was underpowered $(<0.8)$. Second, with a $21 \%$ SNP heritability for AMPA and 5\% for MVPA, the low variance of genetic instruments (AMPA: 0.2\%; MVPA: 0.07\%) may imply that the current discovered SNPs cannot be considered as powerful proxies for PA. Furthermore, although we applied the most up-to-date MR methods, we cannot completely rule out any potential horizontal pleiotropy until we know all biological functions for each SNP. Third, in two-sample MR analysis, weak instrument bias is in the direction of the null while the partial overlapping data between exposure and outcome from UK Biobank may bias against the null. ${ }^{29}$ However, the sensitivity analysis by removing UK Biobank participants from the colorectal cancer GWAS broadens the $\mathrm{Cl}$ for association with MVPA while narrowing the $\mathrm{Cl}$ for AMPA slightly. The overall results did not change. Fourth, sBIA is not a perfect method to measure body fat distribution. However, the correlation between body fat measured by $\mathrm{SBIA}$ and MRI is $\sim 0.8^{58}$ and there is no available GWAS with enough power on MRI or DEXA measured body fat distribution or body fat percentage available. Fifth, because we do not have access to individual-level data we did not perform stratified analysis by gender or tumour site, even though evidence from a European multinational cohort study showed that PA was associated with proximal colon cancer and distal colon cancer risk but not with rectal cancer risk. ${ }^{59}$ Hence, we may have underestimated the effect of PA on colonic cancer risk in our dataset by including rectal cancer. Finally, all analyses were performed using instrumental variables and summary level data 
derived from the GWAS on individuals of European ancestry, which may impact the generalisability of our findings to nonEuropean populations.

\section{CONCLUSIONS}

The results of this study establish a causal role of both subjectively and objectively measured PA in colorectal cancer risk, independent of the obesity and body fat distribution. Our results suggest that promoting and facilitating exercise could result in a decrease in colorectal cancer incidence, regardless of individuals' weight or body fat distribution.

\section{ACKNOWLEDGEMENTS}

We acknowledge the excellent technical support from Stuart Reid. We are grateful to Donna Markie and all those who continue to contribute to recruitment, data collection and data curation for the Study of Colorectal Cancer in Scotland studies. We acknowledge that these studies would not be possible without the patients and surgeons who took part. We acknowledge the expert support on sample preparation from the Genetics Core of the Edinburgh Welcome Trust Clinical Research Facility. This research has been conducted using the UK Biobank Resource under Application Number 7441.

\section{AUTHOR CONTRIBUTIONS}

M.G.D., M.T. and E.T. conceived this study. X.Z., M.T., E.T., J.M.B.R., S.B. and X.L. designed the methodology. S.M.F., M.G.D., H.C., P.J.L., P.B., M.W., R.S.H., I.P.M.T. contributed to the generation of colorectal cancer GWASs and data preparation. Y.C.K. contributed to the generation of physical activity GWAS and data preparation. X.Z. conducted data analysis with the supervision from M.T. and E.T. X.Z. drafted the manuscript, conducted data interpretation with E.T., M.T. and M.G.D. X.L., S.B., Y.C.K., S.M.F., P.J.L., P.B., M.W., J.M.B.R., R.S.H., I.P.M.T. and H.C. contributed to the results interpretation and manuscript drafting and revision.

\section{ADDITIONAL INFORMATION}

Ethics approval and consent to participate All included datasets were approved by respective ethics/institutional review committees, in accordance with the Declaration of Helsinki. The details were described in Law et al. ${ }^{14}$.

\section{Consent to publish Not applicable.}

Data availability Availability of colorectal cancer GWASs was described by Law et al. ${ }^{14}$ (PMID 31089142). Availability of self-reported habitual physical activity GWAS was described by Klimentidis et al. ${ }^{15}$ (PMID 29899525). The summary-level data from the average acceleration vector magnitude GWAS was available at Doherty et al. ${ }^{16}$ (PMID 30531941). The summary-level data for GWAS on BMI, body fat percentage, waist circumference and body fat distribution were extracted from Locke et al. ${ }^{60}$ (PMID 25673413), Bycroft et al. ${ }^{61}$ (PMID 30305743), Shungin et al. ${ }^{62}$ (PMID 25673412) and Ralk-Andersen et al. ${ }^{11}$ (PMID 30664634), respectively. All data generated during this study are included in this published article and its supplementary information files.

Competing interests The authors declare no competing interests.

Funding information This work was supported by Cancer Research UK programme grant [grant number C348/A18927]; Cancer Research UK [grant number C1298/ A25514]; National Cancer Research Network; Cancer Research UK Career Development Fellowship [grant number C31250/A22804]; The Darwin Trust of Edinburgh; National Institute of Health [grant number R01 HL136528].

Supplementary information is available for this paper at https://doi.org/10.1038/ s41416-020-01236-2.

Note This work is published under the standard license to publish agreement. After 12 months the work will become freely available and the license terms will switch to a Creative Commons Attribution 4.0 International (CC BY 4.0).

Publisher's note Springer Nature remains neutral with regard to jurisdictional claims in published maps and institutional affiliations.

\section{REFERENCES}

1. Bray, F., Ferlay, J., Soerjomataram, I., Siegel, R. L., Torre, L. A. \& Jemal, A. Global cancer statistics 2018: GLOBOCAN estimates of incidence and mortality worldwide for 36 cancers in 185 countries. CA Cancer J. Clin. 68, 394-424 (2018).

2. Bardou, M., Barkun, A. N. \& Martel, M. Obesity and colorectal cancer. Gut 62 , 933-947 (2013).

3. WHO. Physical activity and adults. http://www.who.int/dietphysicalactivity/ factsheet_adults/en/ (2018).

4. Rezende, L. F. M., Sa, T. H., Markozannes, G., Rey-Lopez, J. P., Lee, I. M., Tsilidis, K. K. et al. Physical activity and cancer: an umbrella review of the literature including 22 major anatomical sites and 770000 cancer cases. Br. J. Sports Med. 52, 826-833 (2018)

5. Mahmood, S., MacInnis, R. J., English, D. R., Karahalios, A. \& Lynch, B. M. Domainspecific physical activity and sedentary behaviour in relation to colon and rectal cancer risk: a systematic review and meta-analysis. Int. J. Epidemiol. 46, 1797-1813 (2017).

6. Mahmood, S., Nguyen, N. H., Bassett, J. K., MacInnis, R. J., Karahalios, A., Owen, N. et al. A quantitative bias analysis to estimate measurement error-related attenuation of the association between self-reported physical activity and colorectal cancer risk. Int. J. Epidemiol. 49, 153-161 (2019).

7. Didelez, V. \& Sheehan, N. Mendelian randomization as an instrumental variable approach to causal inference. Stat. Methods Med. Res. 16, 309-330 (2007).

8. Papadimitriou, N., Dimou, N., Tsilidis, K. K., Banbury, B., Martin, R. M., Lewis, S. J. et al. Physical activity and risks of breast and colorectal cancer: a Mendelian randomisation analysis. Nat. Commun. 11, 597 (2020).

9. Gallagher, D., Heymsfield, S. B., Heo, M., Jebb, S. A., Murgatroyd, P. R. \& Sakamoto, Y. Healthy percentage body fat ranges: an approach for developing guidelines based on body mass index. Am. J. Clin. Nutr. 72, 694-701 (2000).

10. Thomas, E. L., Frost, G., Taylor-Robinson, S. D. \& Bell, J. D. Excess body fat in obese and normal-weight subjects. Nutr. Res. Rev. 25, 150-161 (2012).

11. Rask-Andersen, M., Karlsson, T., Ek, W. E. \& Johansson, A. Genome-wide association study of body fat distribution identifies adiposity loci and sex-specific genetic effects. Nat. Commun. 10, 339 (2019).

12. Cornish, A. J., Law, P. J., Timofeeva, M., Palin, K., Farrington, S. M., Palles, C. et al. Modifiable pathways for colorectal cancer: a mendelian randomisation analysis. Lancet Gastroenterol. Hepatol. 5, 55-62 (2020).

13. Vithayathil, M., Carter, P., Kar, S., Mason, A. M., Burgess, S. \& Larsson, S. C. Body size and composition and site-specific cancers in UK Biobank: a Mendelian randomisation study. Preprint at https://biorxiv.org/10.1101/2020.02.28.970459 (2020).

14. Law, P. J., Timofeeva, M., Fernandez-Rozadilla, C., Broderick, P., Studd, J., Fernandez-Tajes, J. et al. Association analyses identify 31 new risk loci for colorectal cancer susceptibility. Nat. Commun. 10, 2154 (2019).

15. Klimentidis, Y. C., Raichlen, D. A., Bea, J., Garcia, D. O., Wineinger, N. E., Mandarino, L. J. et al. Genome-wide association study of habitual physical activity in over 377,000 UK Biobank participants identifies multiple variants including CADM2 and APOE. Int. J. Obes. 42, 1161-1176 (2018).

16. Doherty, A., Smith-Byrne, K., Ferreira, T., Holmes, M. V., Holmes, C., Pulit, S. L. et al. GWAS identifies 14 loci for device-measured physical activity and sleep duration. Nat. Commun. 9, 5257 (2018).

17. Sudlow, C., Gallacher, J., Allen, N., Beral, V., Burton, P., Danesh, J. et al. UK Biobank: an open access resource for identifying the causes of a wide range of complex diseases of middle and old age. PLoS Med. 12, e1001779 (2015).

18. Doherty, A., Jackson, D., Hammerla, N., Plotz, T., Olivier, P., Granat, M. H. et al. Large scale population assessment of physical activity using wrist worn accelerometers: the UK Biobank Study. PLoS ONE 12, e0169649 (2017).

19. Burgess, S., Scott, R. A., Timpson, N. J., Davey Smith, G., Thompson, S. G. \& Consortium, E.-l. Using published data in Mendelian randomization: a blueprint for efficient identification of causal risk factors. Eur. J. Epidemiol. 30, 543-552 (2015).

20. Higgins, J. \& Green, S. In Cochrane Handbook for Systematic Reviews of Interventions Version 5.1.0 (eds Jonathan, J., Higgins, J. \& Douglas, G.) 28 (The Cochrane Collaboration, 2011).

21. Burgess, S. \& Thompson, S. G. Multivariable Mendelian randomization: the use of pleiotropic genetic variants to estimate causal effects. Am. J. Epidemiol. 181, 251-260 (2015).

22. Slob, E. A. W. \& Burgess, S. A comparison of robust Mendelian randomization methods using summary data. Genet. Epidemiol. 44, 313-329 (2020).

23. Verbanck, M., Chen, C.-Y., Neale, B. \& Do, R. Detection of widespread horizontal pleiotropy in causal relationships inferred from Mendelian randomization between complex traits and diseases. Nat. Genet. 50, 693 (2018).

24. Rees, J. M., Wood, A. M., Dudbridge, F., Burgess, S. Robust methods in Mendelian randomization via penalization of heterogeneous causal estimates. PloS one. 14, e0222362 (2019). 
25. Bowden, J., Davey Smith, G. \& Burgess, S. Mendelian randomization with invalid instruments: effect estimation and bias detection through Egger regression. Int. J. Epidemiol. 44, 512-525 (2015).

26. Hemani, G., Zheng, J., Elsworth, B., Wade, K. H., Haberland, V., Baird, D. et al. The MR-Base platform supports systematic causal inference across the human phenome. Elife 7, e34408 (2018).

27. Hartwig, F. P., Davey Smith, G. \& Bowden, J. Robust inference in summary data Mendelian randomization via the zero modal pleiotropy assumption. Int. J. Epidemiol. 46, 1985-1998 (2017).

28. Bowden, J., Davey, Smith, G., Haycock, P. C. \& Burgess, S. Consistent estimation in Mendelian randomization with some invalid instruments using a weighted median estimator. Genet. Epidemiol. 40, 304-314 (2016).

29. Burgess, S., Davies, N. M. \& Thompson, S. G. Bias due to participant overlap in two-sample Mendelian randomization. Genet. Epidemiol. 40, 597-608 (2016).

30. Welter, D., MacArthur, J., Morales, J., Burdett, T., Hall, P., Junkins, H. et al. The NHGRI GWAS Catalog, a curated resource of SNP-trait associations. Nucleic Acids Res. 42, D1001-D1006 (2013).

31. MacArthur, J., Bowler, E., Cerezo, M., Gil, L., Hall, P., Hastings, E. et al. The new NHGRI-EBI Catalog of published genome-wide association studies (GWAS Catalog). Nucleic Acids Res. 45, D896-D901 (2017).

32. Staley, J. R., Blackshaw, J., Kamat, M. A., Ellis, S., Surendran, P., Sun, B. B. et al. PhenoScanner: a database of human genotype-phenotype associations. Bioinformatics 32, 3207-3209 (2016).

33. Kamat, M. A., Blackshaw, J. A., Young, R., Surendran, P., Burgess, S., Danesh, J. et al. PhenoScanner V2: an expanded tool for searching human genotype-phenotype associations. Bioinformatics 35, 4851-4853 (2019).

34. Yavorska, O. O. \& Burgess, S. MendelianRandomization: an R package for performing Mendelian randomization analyses using summarized data. Int. J. Epidemiol. 46, 1734-1739 (2017).

35. Brion, M. J., Shakhbazov, K. \& Visscher, P. M. Calculating statistical power in Mendelian randomization studies. Int. J. Epidemiol. 42, 1497-1501 (2013).

36. Shim, H., Chasman, D. I., Smith, J. D., Mora, S., Ridker, P. M., Nickerson, D. A. et al. A multivariate genome-wide association analysis of $10 \mathrm{LDL}$ subfractions, and their response to statin treatment, in 1868 Caucasians. PLoS one 10, e0120758 (2015).

37. Burgess, S., Thompson, S. G. \& CRP CHD Genetics Collaboration. Avoiding bias from weak instruments in Mendelian randomization studies. Int. J. Epidemiol. 40, 755-764 (2011).

38. Ashford, J. W. APOE genotype effects on Alzheimer's disease onset and epidemiology. J. Mol. Neurosci. 23, 157-165 (2004).

39. Eichner, J. E., Dunn, S. T., Perveen, G., Thompson, D. M., Stewart, K. E. \& Stroehla, B. C. Apolipoprotein E polymorphism and cardiovascular disease: a HuGE review. Am. J. Epidemiol. 155, 487-495 (2002).

40. Wolin, K. Y., Yan, Y., Colditz, G. A. \& Lee, I. M. Physical activity and colon cancer prevention: a meta-analysis. Br. J. Cancer 100, 611-616 (2009).

41. Park, S. Y., Wilkens, L. R., Haiman, C. A. \& Le Marchand, L. Physical activity and colorectal cancer risk by sex, race/ethnicity, and subsite: the Multiethnic Cohort Study. Cancer Prev. Res. 12, 315-326 (2019).

42. World Cancer Research Fund American/Institute for Cancer Research. Continuous update project expert report. Physical Activity and the Risk of Cancer www. dietandcancerreport.org (2018).

43. Shaw, E., Farris, M. S., Stone, C. R., Derksen, J. W. G., Johnson, R., Hilsden, R. J. et al. Effects of physical activity on colorectal cancer risk among family history and body mass index subgroups: a systematic review and meta-analysis. BMC Cancer 18, 71 (2018).

44. Ainsworth, B. E., Haskell, W. L., Herrmann, S. D., Meckes, N., Bassett, D. R. Jr, TudorLocke, C. et al. 2011 Compendium of physical activities: a second update of codes and MET values. Med. Sci. Sports Exerc. 43, 1575-1581 (2011).

45. Dyrstad, S. M., Hansen, B. H., Holme, I. M. \& Anderssen, S. A. Comparison of selfreported versus accelerometer-measured physical activity. Med. Sci. Sports Exerc. 46, 99-106 (2014)

46. Carter, A. R., Sanderson, E., Hammerton, G., Richmond, R., Smith, G. D., Heron, J. et al. Mendelian randomisation for mediation analysis: current methods and challenges for implementation. Preprint at https://biorxiv.org/10.1101/835819 (2019).

47. Cordain, L., Latin, R. W. \& Behnke, J. J. The effects of an aerobic running program on bowel transit time. J. Sports Med. Phys. Fit. 26, 101-104 (1986).

48. Romeo, J., Warnberg, J., Pozo, T. \& Marcos, A. Physical activity, immunity and infection. Proc. Nutr. Soc. 69, 390-399 (2010).

49. Balkau, B., Mhamdi, L., Oppert, J.-M., Nolan, J., Golay, A., Porcellati, F. et al. Physical activity and insulin sensitivity the RISC study. Diabetes 57, 2613-2618 (2008).

50. Martinez, M. E., Heddens, D., Earnest, D. L., Bogert, C. L., Roe, D., Einspahr, J. et al. Physical activity, body mass index, and prostaglandin E2 levels in rectal mucosa. J. Natl Cancer Inst. 91, 950-953 (1999).

51. Bartram, H. P. \& Wynder, E. L. Physical activity and colon cancer risk? Physiological considerations. Am. J. Gastroenterol. 84, 109-112 (1989).

52. Nakanishi, M. \& Rosenberg, D. W. Multifaceted roles of PGE2 in inflammation and cancer. Semin. Immunopathol. 35, 123-137 (2013).

53. Wertheim, B. C., Martínez, M. E., Ashbeck, E. L., Roe, D. J., Jacobs, E. T., Alberts, D. S. et al. Physical activity as a determinant of fecal bile acid levels. Cancer Epidemiol. Biomark. Prev. 18, 1591-1598 (2009).

54. Colley, R. C., Butler, G., Garriguet, D., Prince, S. A. \& Roberts, K. C. Comparison of self-reported and accelerometer-measured physical activity in Canadian adults. Health Rep. 29, 3-15 (2018).

55. White, T., Westgate, K., Wareham, N. J. \& Brage, S. Estimation of physical activity energy expenditure during free-living from wrist accelerometry in UK adults. PLOS ONE 11, e0167472 (2016).

56. Goode, A. P., Hall, K. S., Batch, B. C., Huffman, K. M., Hastings, S. N., Allen, K. D. et al. The impact of interventions that integrate accelerometers on physical activity and weight loss: a systematic review. Ann. Behav. Med. 51, 79-93 (2017).

57. Jarvis, D., Mitchell, J. S., Law, P. J., Palin, K., Tuupanen, S., Gylfe, A. et al. Mendelian randomisation analysis strongly implicates adiposity with risk of developing colorectal cancer. Br. J. Cancer 115, 266-272 (2016).

58. Borga, M., West, J., Bell, J. D., Harvey, N. C., Romu, T., Heymsfield, S. B. et al. Advanced body composition assessment: from body mass index to body composition profiling. J. Investig. Med. 66, 1-9 (2018).

59. Murphy, N., Ward, H. A., Jenab, M., Rothwell, J. A., Boutron-Ruault, M. C., Carbonnel, F. et al. Heterogeneity of colorectal cancer risk factors by anatomical subsite in 10 European countries: a multinational cohort study. Clin. Gastroenterol. Hepatol. 17, 1323-31 e1326 (2019).

60. Locke, A. E., Kahali, B., Berndt, S. I., Justice, A. E., Pers, T. H., Day, F. R. et al. Genetic studies of body mass index yield new insights for obesity biology. Nature. 518, 197-206 (2015).

61. Bycroft, C., Freeman, C., Petkova, D., Band, G., Elliott, L. T., Sharp, K. et al. The UK Biobank resource with deep phenotyping and genomic data. Nature. 562, 203-209 (2018).

62. Shungin, D., Winkler, T. W., Croteau-Chonka, D. C., Ferreira, T., Locke, A. E., Mägi, R. et al. New genetic loci link adipose and insulin biology to body fat distribution. Nature. 518, 187-196 (2015).

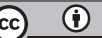

Open Access This article is licensed under a Creative Commons Attribution 4.0 International License, which permits use, sharing, adaptation, distribution and reproduction in any medium or format, as long as you give appropriate credit to the original author(s) and the source, provide a link to the Creative Commons licence, and indicate if changes were made. The images or other third party material in this article are included in the article's Creative Commons licence, unless indicated otherwise in a credit line to the material. If material is not included in the article's Creative Commons licence and your intended use is not permitted by statutory regulation or exceeds the permitted use, you will need to obtain permission directly from the copyright holder. To view a copy of this licence, visit http://creativecommons. org/licenses/by/4.0/.

(c) The Author(s) 2021 